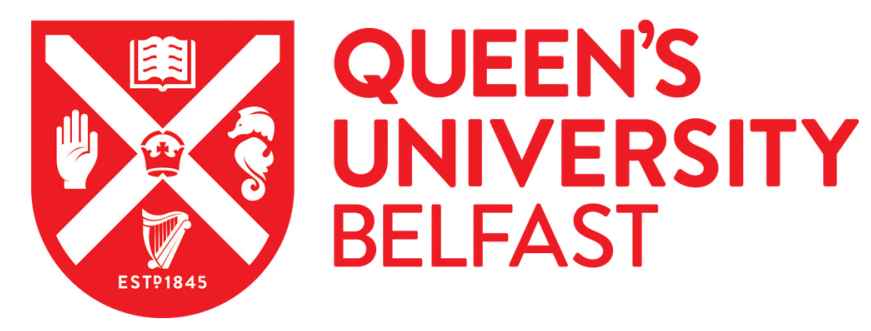

\title{
Equivalent Circuit Model of Twisted Split Ring Frequency Selective Surfaces
}

Zelenchuk, D., \& Fusco, V. (2016). Equivalent Circuit Model of Twisted Split Ring Frequency Selective Surfaces. In Proceedings of 10th European Conference on Antennas \& Propagation (EUCAP 2016) Institute of Electrical and Electronics Engineers Inc..

Published in:

Proceedings of 10th European Conference on Antennas \& Propagation (EUCAP 2016)

Document Version:

Peer reviewed version

Queen's University Belfast - Research Portal:

Link to publication record in Queen's University Belfast Research Portal

\section{Publisher rights}

( $) 2016$ IEEE. Personal use of this material is permitted. Permission from IEEE must be obtained for all other uses, in any current or future media, including reprinting/republishing this material for advertising or promotional purposes, creating new collective works, for resale or redistribution to servers or lists, or reuse of any copyrighted component of this work in other works

\section{General rights}

Copyright for the publications made accessible via the Queen's University Belfast Research Portal is retained by the author(s) and / or other copyright owners and it is a condition of accessing these publications that users recognise and abide by the legal requirements associated with these rights.

Take down policy

The Research Portal is Queen's institutional repository that provides access to Queen's research output. Every effort has been made to ensure that content in the Research Portal does not infringe any person's rights, or applicable UK laws. If you discover content in the Research Portal that you believe breaches copyright or violates any law, please contact openaccess@qub.ac.uk. 


\title{
Equivalent Circuit Model of Twisted Split Ring Frequency Selective Surfaces
}

\author{
Dmitry Zelenchuk ${ }^{1}$, Vincent Fusco ${ }^{1}$ \\ ${ }^{1}$ ECIT, Queens University of Belfast, Belfast, United Kingdom, d.zelenchuk@qub.ac.uk
}

\begin{abstract}
This paper proposes a wideband equivalent circuit model for a twisted split ring frequency selective surface (FSS). Such surfaces can be used for modelling and design of polarisation sensitive surfaces such as circularly polarized selective surfaces as well as structures with asymmetric transmission. The proposed model is based extraction of equivalent circuit parameters from a single split ring (SRR) FSS and magnetic coupling from periodic eigenmode analysis of the coupled SRR. The resulting equivalent circuit model demonstrates excellent agreement with full-wave simulations.

Index Terms-frequency selective surfaces, polarisation transformation, split ring resonator, near-field coupling, equivalent circuit model.
\end{abstract}

\section{INTRODUCTION}

Recent advances in the research of frequency selective surfaces (FSS) and metamaterials have demonstrated novel properties of dichroism such as asymmetric transmission, selective transmission of a particular polarization or polarization rotation [1]-[5].

A geometry of particular interest is a frequency selective surface consisting of coupled twisted split ring resonators (SRR) [6]-[8]. Such structures were demonstrated to exhibit the polarization transformation properties mentioned above as well as spectral selectivity [9] and wave front transformation [10] These structures are easy to manufacture as there are no vias required to ensure chiral properties of the structures [2].

The unit cell of such a structure is shown in Fig. 1. Previously reported models described the behaviour of an SRR as a linearly polarised dipole-like particle in the vicinity of resonance and considered near field coupling properties only.

Below we propose a new wideband equivalent circuit model for frequency selective surfaces composed of coupled SRR that takes into account both co-polar and cross-polar scattering of individual SRRs as well as the near-field coupling between them.

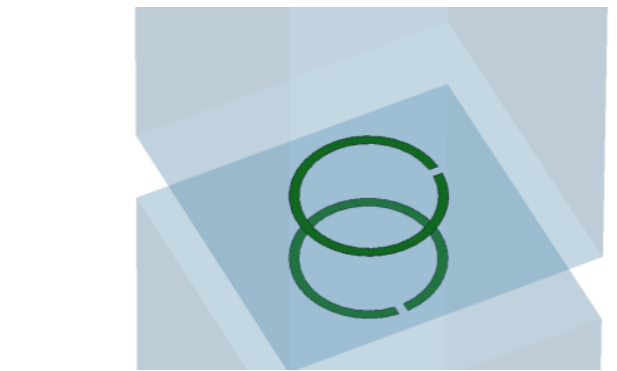

Fig. 1. Unit cell of a twisted split ring FSS.

\section{THEORY}

\section{A. Single SRR FSS}

Let us first analyse a single SRR FSS. The structure resonates when the strip length is close to half-wavelength. The equivalent circuit of the single SSR is present in Fig. 2. The circuit parameters can be easily extracted from values of reflection coefficients by fitting equation (1) to a full-wave simulation.

$$
\begin{aligned}
& \Gamma(\omega)=-\frac{1}{1+2 Z_{c} / Z_{0}} \\
& Z_{c}(\omega)=\frac{1-\omega^{2} L_{c} C_{c}}{j \omega C_{c}+j \omega C_{p}\left(1-\omega^{2} L_{c} C_{c}\right)}
\end{aligned}
$$

A single SRR FSS with period $\mathrm{d}=35 \mathrm{~mm}$, width $\mathrm{w}=1 \mathrm{~mm}$, radius $\mathrm{r}=8 \mathrm{~mm}$ and gap width $\mathrm{c}=.1 \mathrm{~mm}$ has been simulated with CST Microwave Studio under linearly polarized excitation parallel to the gap at normal incidence. This excites single SRR fundamental resonance.

By applying the extraction process described above it has been found that $L_{c}=251 \mathrm{nH}, C_{c}=0.0109 \mathrm{pF}$ and $C_{p}=$ $0.0095 p F$. One can see in Fig. 3 that the fitted curve closely follows the simulated one. It has to be noted that if the additional capacitance $C_{p}$ is omitted then the fitting process works only in the vicinity of the resonance. 


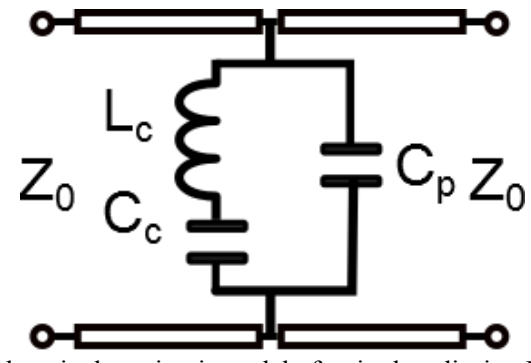

Fig. 2. Wideband equivalent circuit model of a single split ring FSS.

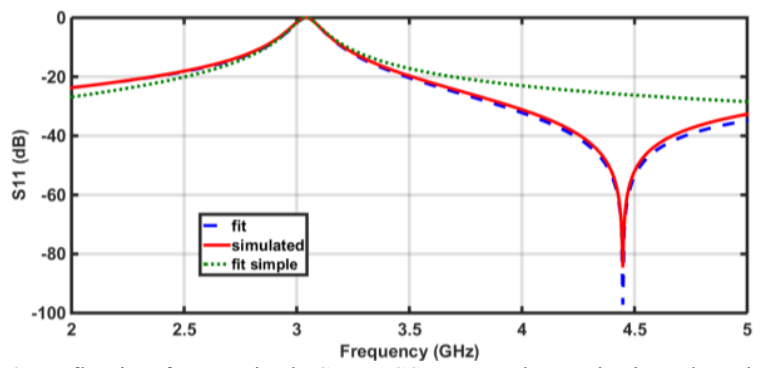

Fig. 3. Reflection from a single SRR FSS at co-polar excitation: dotted greensimple LC model with $C_{p}=0 p F$; dashed- blue $-\mathrm{LC}$ with with $C_{p}=$ $0.0095 p F$ and solid red-CST Microwave Studio.

When the incident electric field is orthogonal to the gap, the split ring resonates at a higher frequency and can be modelled by a simple LC- resonator in Fig. 2 with $C_{p}=0 p F$. The fitted parameters were found as $L_{c}=30.15 \mathrm{nH}, C_{c}=0.0167 \mathrm{pF}$. As one can see in Fig. 4 the fitted curve is in very good agreement with the full-wave simulation.

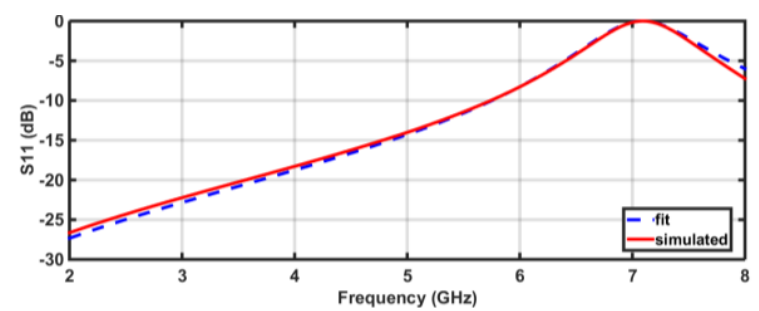

Fig. 4. Reflection from a single SRR FSS at cross-polar excitation:; dashedblue - simple LC model with $C_{p}=0 p F$ and solid red - CST Microwave Studio.

\section{B. Coupled SRR FSS}

A pair of split-ring resonators separated by an air-substrate as shown in Fig. 1 exhibits even and odd resonances with the currents in both SRR excited in phase or in anti-phase, see Fig. 5.

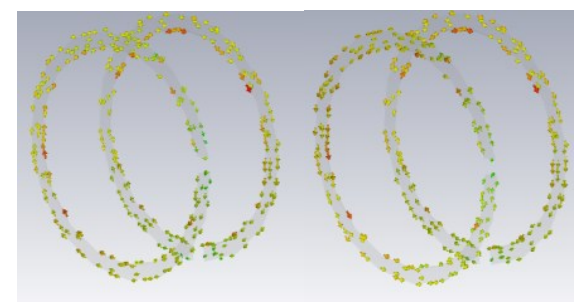

(a)

(b)

Fig. 5. Surface current distribution for a $90^{\circ}$ twisted SRR at $\mathrm{h}=6 \mathrm{~mm}$ : (a): even mode; (b) odd mode.
It has been shown in [6] that the even and odd mode resonant frequencies can determined from a Lagrangian model as follows.

$$
\begin{aligned}
& \omega_{o}=\omega_{0} \sqrt{\frac{1-\widetilde{k_{e}}}{1-k_{h}}} \\
& \omega_{e}=\omega_{0} \sqrt{\frac{1+\widetilde{k_{e}}}{1+k_{h}}}
\end{aligned}
$$

where $\omega_{0}$ is the resonant frequency of a single SRR, $\widetilde{k_{e}}=$ $k_{e}\left(\cos \theta+a \cos ^{2} \theta+b \cos ^{3} \theta\right), k_{h}$ is the magnetic coupling and $k_{e}$ is electric coupling coefficient, $\theta$ is the twist angle, $a$ and $b$ are fitting coefficients related to higher-order multipole expansion. It has to be noted that the magnetic coupling is isotropic and does not depend on the twist angle $\theta$ [11].

The eigenmode solver in CST Microwave studio with upper and lower $\mathrm{z}$-boundaries set at $10 \mathrm{~mm}$ and lateral periodic boundary phase shift set at $\varphi_{x}=0, \varphi_{y}=0$ for the structure above with $\mathrm{h}=6 \mathrm{~mm}$ was deployed The resonant frequencies of the first two modes are plotted in Fig. 6. These results are used to obtain the coupling coefficients from the dispersion curves by curve fitting. In order to get robust results this fitting was performed on the mixed coupling coefficient defined as

$$
k_{m}=\frac{\omega_{e}^{2}-\omega_{o}^{2}}{\omega_{e}^{2}+\omega_{o}^{2}}=\frac{k_{h}-\widetilde{k_{e}}}{1-k_{h} \widetilde{k_{e}}}
$$

The results of the fitting process are demonstrated Fig. 7. The fitted coefficients are $\mathrm{a}=0.215, \mathrm{~b}=0.0957, k_{h}=0.1571$ and $k_{e}=0.0742$. Thus it is apparent that this particular structure is dominated by magnetic coupling.

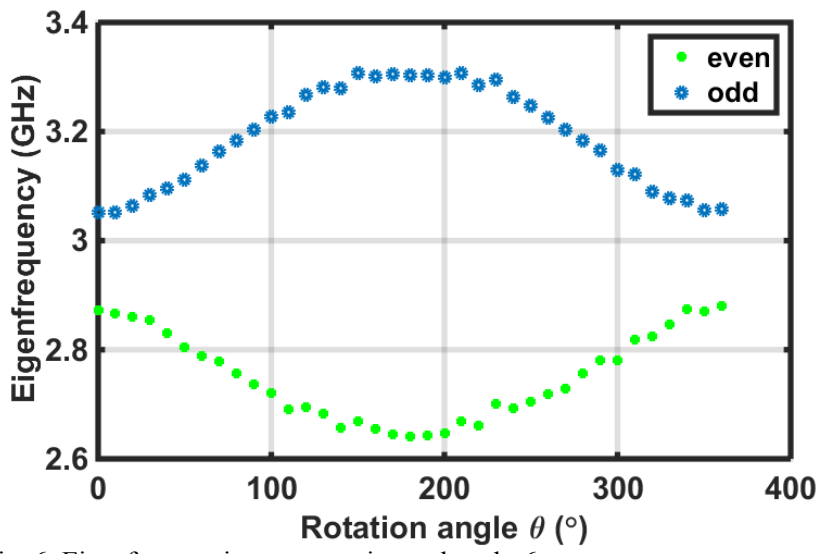

Fig. 6. Eigenfrequencies versus twist angle at $\mathrm{h}=6 \mathrm{~mm}$. 
A particular interest for circularly polarised selectivity present the structure in Fig. 1 with a $90^{\circ}$ twist . As one can see from the above formula for $\widetilde{k_{e}}$, for such a twist the electrical coupling vanishes and only magnetic coupling exists. The eigenmode analysis for such a structure has been performed for various values of $h$ and the results shown in Fig. 7.

Based on these results the dependence of the magnetic coupling over separation distance was fitted as

$$
k_{h}(h)=\frac{-0.083 h+1.792}{h+2.089}
$$

where $h$ is in $\mathrm{mm}$.

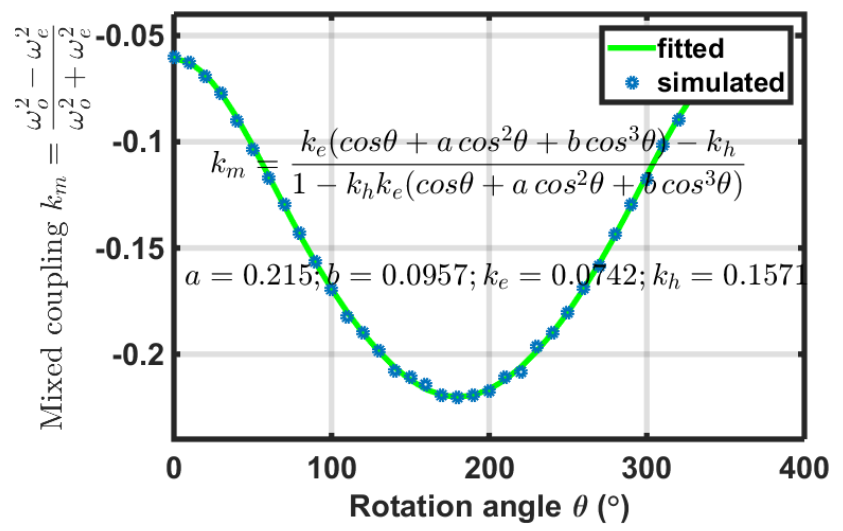

Fig. 7. Mixed coupling versus twist angle for thin rings at $\mathrm{h}=6 \mathrm{~mm}$.

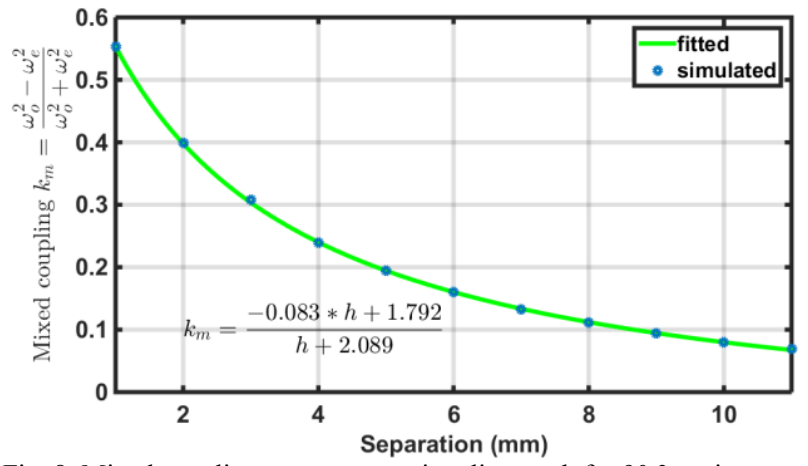

Fig. 8. Mixed coupling versus separation distance $\mathrm{h}$ for $90^{\circ}$ twist.

\section{Coupled SRR FSS transmission line model}

Using the data obtained in the previous sections an equivalent circuit model for the $90^{\circ}$ twisted SRR FSS excited at normal incidence is proposed in Fig. 9. The model is based on a magnetically coupled resonator circuit [12] and augmented with additional LC-resonators representing cross-polar scattering from individual SRRs. In fact the part of the circuit on the left of the $T T^{\prime}$ virtual plane represents a Floquet waveguide with TM-mode and the one on the right a Floquet waveguide with TE-mode.

Port 1 is modelling TE-excitation from the top of the structure, chosen to be co-polar to the first SRR, port 2 is TEport at the bottom of the FSS, port 3 is TM-port at the top of the FSS (co-polar to the second SRR) and port 4 is TM-port at the bottom of the FSS. This means that transmission from TE to TE mode is modelled by $\mathrm{S} 21$ and $\mathrm{S} 12$, for top and bottom excitation respectively, and reflection from TE to TM mode is S31 and S42, for top and bottom excitation respectively, etc.

The full-wave simulation has been compared to the equivalent circuit simulation with the component parameters extracted from single SRR simulations and eigenmode analysis of the coupled SRR as $L_{3}=251 \mathrm{nH}, C_{3}=0.0109 \mathrm{pF}$ and $C_{1}=0.0095 \mathrm{pF}$, and $L_{2}=30.15 \mathrm{nH}, C_{2}=0.0167 \mathrm{pF}$. The transmission lines in Fig. 9 are of length $h$ characterised by free space impedance. The coupling inductance is found after [12] as

$$
L_{m}=k_{h} L_{3}
$$

This approach allows us to model circuits with different $h$ by utilising (4).

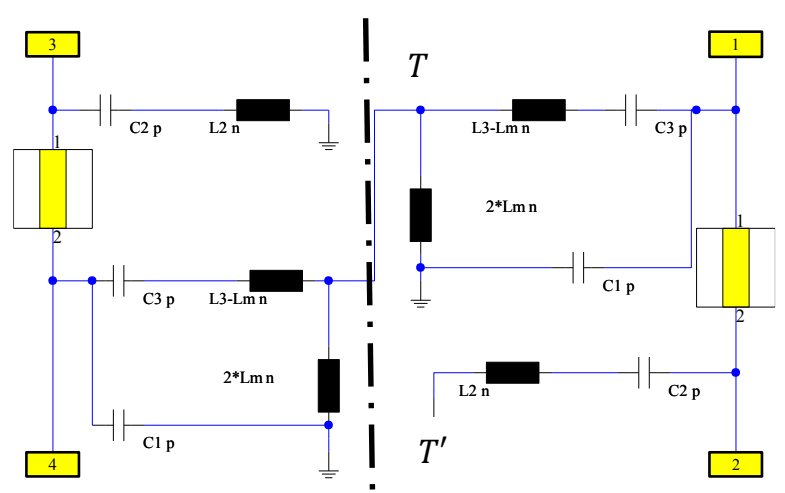

Fig. 9. Equivalent transmission line model for a $90^{\circ}$ twisted SRR FSS.

The results of the comparison are shown in Fig. 10. These demonstrate very good agreement for both magnitude and phase over a wide frequency band.

The proposed model provides an effective tool that accurately describes the twisted SRR FSS behaviour for both cardinal orthogonal linearly polarised excitation possibilities. By simple linear transformation the data can be converted to a circularly polarized basis [13] and thus structures as CP FSS [14] and CPSS [3] could be analysed, as well with asymmetric LP transmission metamaterials [1], [15]. 

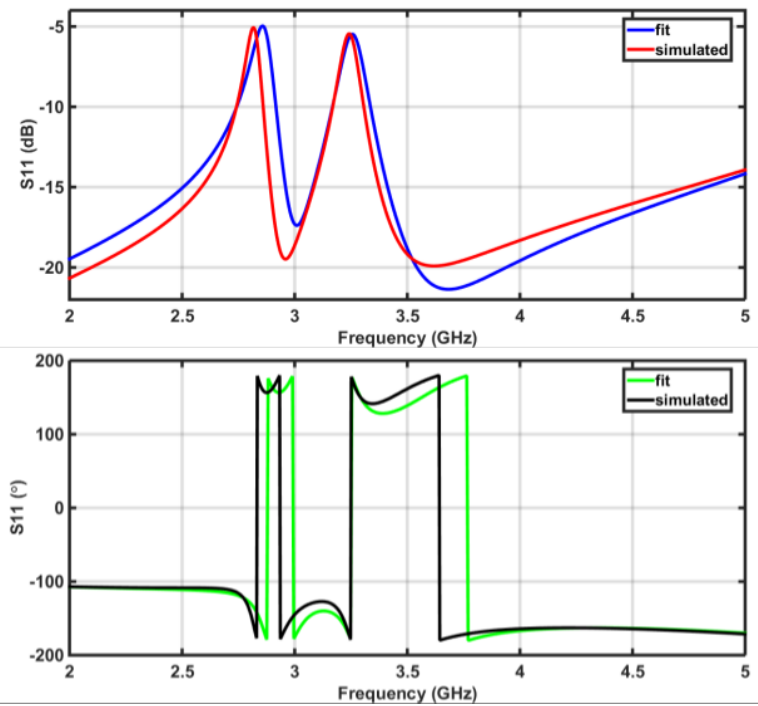

(a)
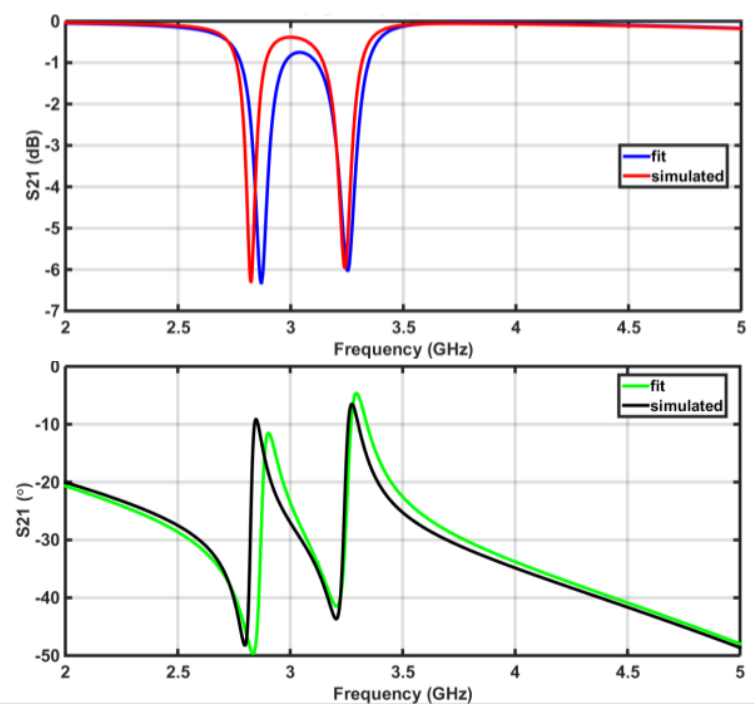

(b)
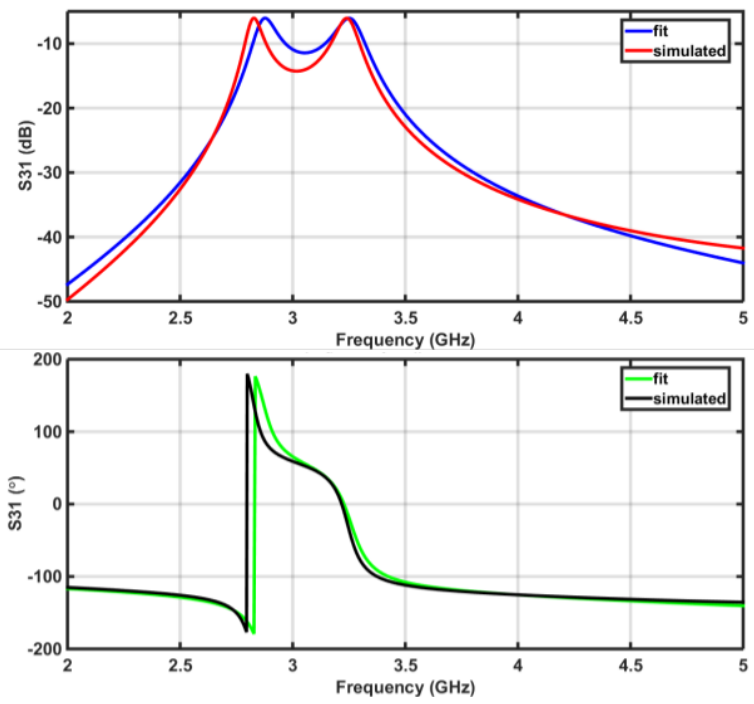

(c)
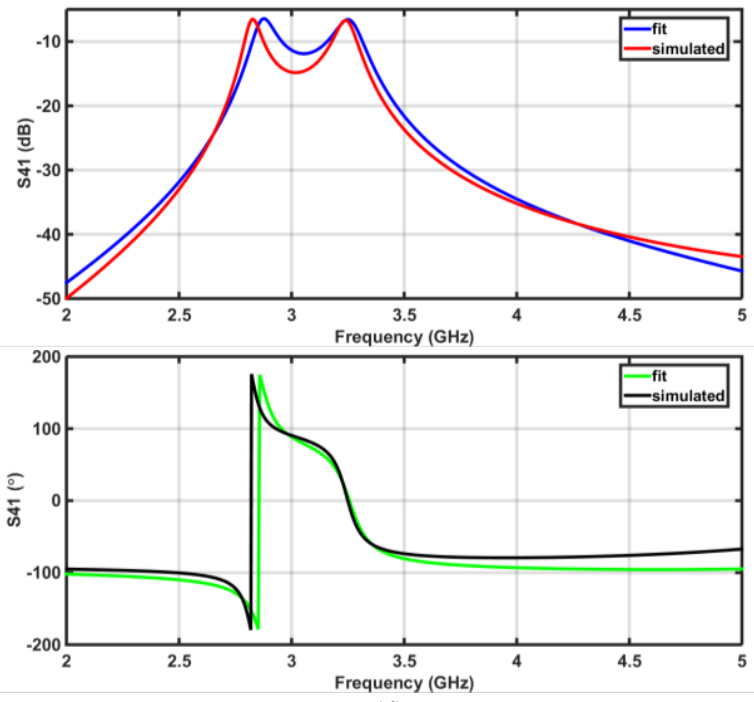

(d)
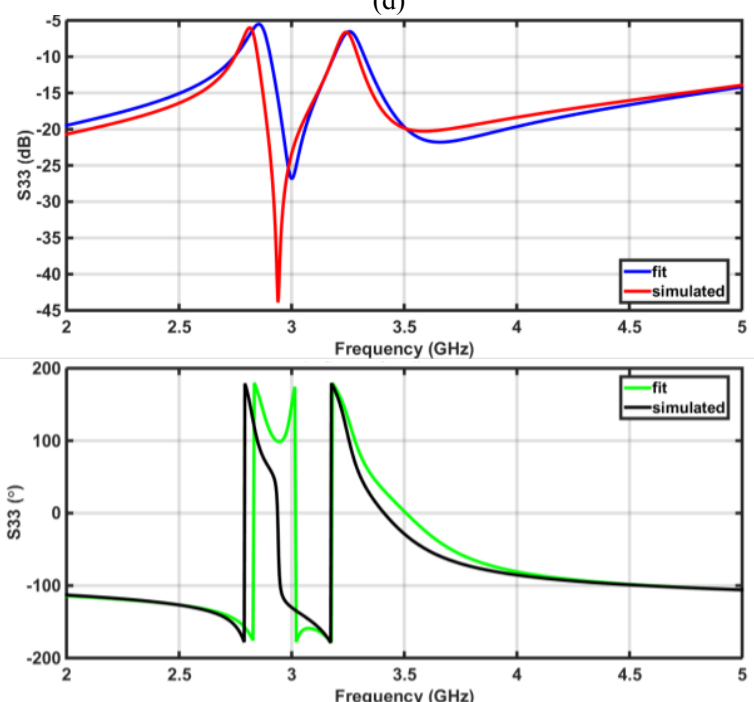

(e)

Fig. 10. Comparison of full-wave simulation with equivalent circuit model for the $90^{\circ}$ twist coupled SRR FSS with separation distance $\mathrm{h}=6 \mathrm{~mm}$ (excitation from the top). (a) - reflection from TE to TE; (b) - transmission from TE to $\mathrm{TE}$, (c) - reflection from TE to TM, (e) - reflection from TM to TM, transmission.

\section{CONCLUSiON}

A new equivalent circuit model for twisted split ring frequency selective surface has been proposed in this paper. The model is capable of modelling stereo SRR behaviour over a much wider band than previously possible. It is based on extraction of equivalent circuit parameters from a single split ring FSS and magnetic coupling from periodic eigenmode analysis and is therefore simple to parameterise from full wave simulation or measurement. The proposed equivalent circuit model demonstrated very good agreement with full-wave simulations performed with CST Microwave Studio and can be used for modelling and design of polarisation sensitive surfaces such as circularly polarized selective surfaces as well as structures with asymmetric transmission. 


\section{REFERENCES}

[1] C. Menzel, C. Helgert, C. Rockstuhl, E. B. Kley, a. Tünnermann, T. Pertsch, and F. Lederer, "Asymmetric transmission of linearly polarized light at optical metamaterials," Phys. Rev. Lett., vol. 104, no. June, pp. 1-4, 2010.

[2] T. Niemi, A. O. Karilainen, and S. a. Tretyakov, "Synthesis of polarization transformers," IEEE Trans. Antennas Propag., vol. 61, no. 6, pp. 3102-3111, 2013.

[3] J. E. Roy and L. Shafai, "Reciprocal circular-polarization-selective surface," IEEE Antennas Propag. Mag., vol. 38, no. 6, pp. 18-32, 1996.

[4] X. Huang, B. Xiao, D. Yang, and H. Yang, "Ultra-broadband $90^{\circ}$ polarization rotator based on bi-anisotropic metamaterial," Opt. Commun., vol. 338, pp. 416-421, 2015.

[5] Y. Cheng, Y. Nie, X. Wang, and R. Gong, "An ultrathin transparent metamaterial polarization transformer based on a twist-split-ring resonator," Appl. Phys. A, vol. 111, no. 1, pp. 209-215, Jan. 2013.

[6] N. Liu, H. Liu, S. Zhu, and H. Giessen, "Stereometamaterials," Nat. Photonics, vol. 3, no. March, pp. 157-162, 2009.

[7] D. a. Powell, K. Hannam, I. V. Shadrivov, and Y. S. Kivshar, "Near-field interaction of twisted split-ring resonators," Phys. Rev. $B$ - Condens. Matter Mater. Phys., vol. 83, pp. 1-6, 2011.

[8] M. Decker, R. Zhao, C. M. Soukoulis, S. Linden, and M. Wegener, "Twisted split-ring-resonator photonic metamaterial with huge optical activity.," Opt. Lett., vol. 35, no. 10, pp. 1593-5, May 2010.
R. Dickie, R. Cahill, V. Fusco, H. S. Gamble, and N. Mitchell, "THz Frequency Selective Surface Filters for Earth Observation Remote Sensing Instruments," IEEE Trans. Terahertz Sci. Technol., vol. 1, pp. 450-461, 2011.

[10] D. Zelenchuk and V. Fusco, "Split Ring FSS Spiral Phase Plate," pp. 1-5, 2012.

[11] H. Liu, J. X. Cao, S. N. Zhu, N. Liu, R. Ameling, and H. Giessen, "Lagrange model for the chiral optical properties of stereometamaterials," Phys. Rev. B - Condens. Matter Mater. Phys., vol. 81, pp. 157-162, 2010

[12] J. Hong and M. Lancaster, Microstrip filters for RF/microwave applications, vol. 7. New York: John Wiley \& Sons, Inc., 2001, pp. $0-471$.

[13] C. Menzel, C. Rockstuhl, and F. Lederer, "Advanced Jones calculus for the classification of periodic metamaterials," Phys. Rev. A, vol. 82, no. 5, p. 053811 , Nov. 2010.

[14] R. Orr, G. Goussetis, V. Fusco, R. Cahill, D. Zelenchuk, A. Pal, E. Saenz, M. Simeoni, and L. Salghetti Droili, "Circular polarisation frequency selective surface operating in $\mathrm{Ku}$ and $\mathrm{Ka}$ band," in Proceedings of the 8th European Conference on Antennas and Propagation (EUCAP), 2014, pp. 2342-2344.

[15] D. Liu, Z. Xiao, X. Ma, L. Wang, K. Xu, J. Tang, and Z. Wang, "Dual-band asymmetric transmission of chiral metamaterial based on complementary U-shaped structure," Appl. Phys. A, vol. 118, pp. 787-791, 2015. 